\title{
A Comparison and Evaluation of Computerized Methods for Blood Vessel Enhancement and Segmentation in Retinal Images
}

\author{
Safia Shabbir, Anam Tariq, and M. Usman Akram
}

\begin{abstract}
Diabetic retinopathy is caused by complications of diabetes, which can eventually lead to blindness. As new blood vessels form at the back of the eye as a part of diabetic retinopathy (DR), they can bleed and blur vision. Detection of these new vessels and their structure in retinal images is very important for diagnosis of diabetic retinopathy. In this paper two different techniques have been compared. First technique uses Gaussian filtering for preprocessing, LoG filtering for enhancement and adaptive thresholding for segmentation purpose. Second technique uses unsharp masking for preprocessing, Gabor wavelet for enhancement and global thresholding for segmentation. The performance of these systems is evaluated on publicly available DRIVE and STARE databases of manually labeled images. Experimental results show that Gabor wavelet method gives best results for vessel enhancement and global threshold gives good results for vessel segmentation in retinal images.
\end{abstract}

Index Terms-Blood vessel, DIABETIC retinopathy (DR), retinal images, unsharp masking, gabor wavelet transform, adaptive thresholding, log filtering, enhancement, segmentation.

\section{INTRODUCTION}

Diabetic retinopathy is a disease which damages the retina and it is caused by the sophistications of diabetes, which leads to blindness. All patients who have diabetes mellitus have the risk of diabetic retinopathy. The risk factor increases with the increase in the span of diabetes. There are no early indications of Diabetic retinopathy. Even some times macular edema does not show any indication rather it creates vision loss rapidly. In retinopathy new blood vessels can be formed at the back of eye and due to their leakage blurred vision is caused. In most cases some blood spots appear on the surface of eye which may disappear after some hours.

A tool which can be used to assist in the diagnosis of diabetic retinopathy should automatically detect all retinal image features such as optic disk, fovea and blood vessel and all abnormalities in retinal image such as microaneurysms hard and soft exudates, hemorrhages and edema.

Computer assisted diagnosis for various diseases is very common now a days and medical imaging is playing a very important part in such computer assisted diagnosis. So, image processing techniques can help in detecting all above mentioned abnormalities from fundus image as they appear

Manuscript received December 18, 2012; revised February 17, 2013.

The authors are with the Department of Software Engineering, Fatima Jinnah Women University, Rawalpindi, Pakistan (e-mail: safia_shabbir@hotmail.com, anam.tariq86@gmail.com, usmakram@gmail.com). with obvious properties (color size and shape).

Most of the vessel segmentation methods are based on watershed algorithm [1], probabilistic filters [2], Hessian based filters [3], improved matched filters [4] and Gabor filter [5]. Methods based on vessel tracking to obtain the vasculature structure, along with vessel diameters and branching points have been proposed by [6] and [7]. Papers [8]-[9] used deformable models for vessels segmentation.

In this paper, we compare the retinal image enhancement techniques that enhance the blood vessels using Gabor wavelet and LoG filtering. We also compare the retinal image vessel segmentation techniques that segment the vascular pattern using global thresholding and adaptive thresholding.

The paper is organized in four sections. In section II, comparison and evaluation of the two techniques for automated vessel enhancement and segmentation is done in detail. Experimental results on the retinal images of the DRIVE and STARE databases and their analysis are given in Section III followed by conclusion in Section IV.

\section{COMPARISON OF BLOOD VESSEl ENHANCEMENT AND SEGMENTATION TECHNIQUES}

The two techniques are compared in this section.

A. Technique Using LoG Filtering and Adaptive
Thresholding

A systematic overview of the first technique is shown in Fig. 1. In summary, given a retinal image, the first step removes the noise from the image; the second step enhances the retinal image making vessels more clear and in the third step segmentation of retinal blood vessels takes place. As a result of these steps, we get a final blood vessels segmented image.

\section{1) Preprocessing}

Preprocessing of an image is done to smooth or enhance the image. Gaussian filter is used to preprocess the image in this technique. Steps for the preprocessing are as follows:

1) Convert image to gray scale image

2) Apply Gaussian blur filter (low pass filter) on gray scale image to smooth the retinal image. (1) Shows the Gaussian blur filter where $\sigma=5$.

$$
G(x, y)=\frac{1}{2 \pi \sigma^{2}} e^{-\frac{x^{2}+y^{2}}{2 \sigma^{2}}}
$$

Fig. 2 shows the original retinal image and preprocessed image. 


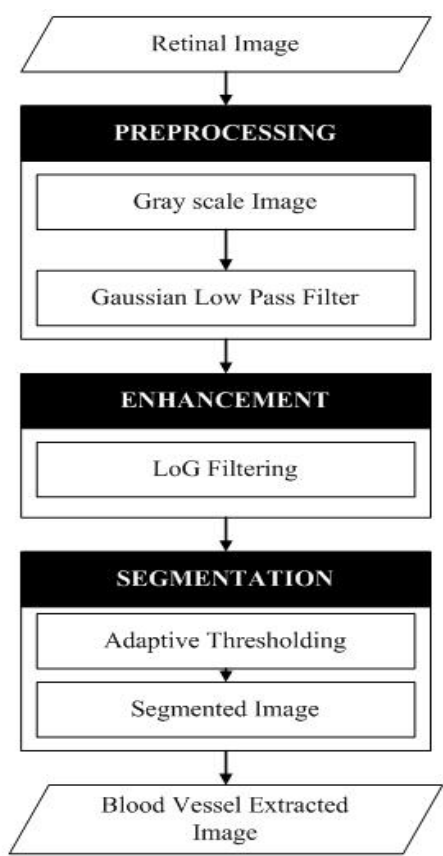

Fig. 1. Flow chart of LoG filtering and adaptive thresholding technique

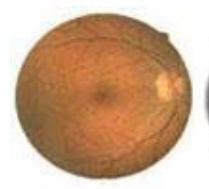

(a)

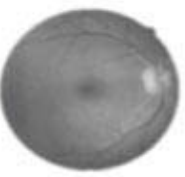

(b)

Fig. 2. Preprocessing: (a) original image (b) gaussian filtered image

\section{2) Blood vessel enhancement}

LoG filtering is used for blood vessel enhancement in this technique. Laplacian filters are basically derivative filters which are used to find edges in images. But the image should be smoothed first before applying laplacian as derivative filters are very sensitive to noise. This two-step process is called Laplacian of Gaussian (LoG) operation. (2) shows the LoG filter where $\sigma=2$.

$$
\operatorname{LoG}(x, y)=\frac{1}{\pi \sigma^{4}}\left[1-\frac{x^{2}+y^{2}}{2 \sigma^{2}}\right] e^{-\frac{x^{2}+y^{2}}{2 \sigma^{2}}}
$$

Fig. 3 shows the preprocessed retinal image and LoG filtered image.

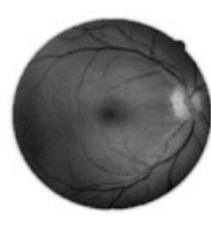

(a)

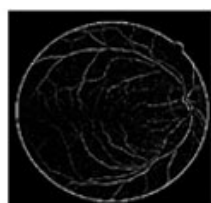

(b)
Fig. 3. Enhancement: (a) preprocessed image (b) LoG filtered image

\section{3) Blood vessel segmentation}

This technique uses adaptive thresholding for segmentation process. The steps of applying adaptive thresholding are:

1) Take the LoG filtered enhanced image.

2) Select a region of interest (ROI) of $95 \times 95$ in the image.

3) Select threshold value for that ROI using the average gray level.

4) Convert this region to binary using the threshold value.

5) Go to step 2 until whole image is segmented.
Fig. 4 shows the LoG filtered image and adaptive threshold image.

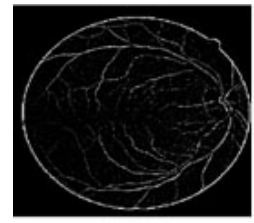

(a)

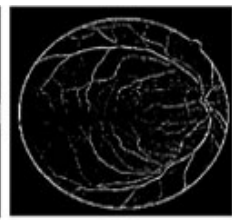

(b)
Fig. 4. Segmentation: (a) LoG filtered image (b) Adaptive thresholded image

\section{B. Technique Using Gabor Wavelet and Global Thresholding}

A systematic overview of the second technique is shown in Fig. 5. In summary, given a retinal image, the first step removes the noise and sharpens the image; the second step enhances the retinal image making vessels more clear and in the third step segmentation of retinal blood vessels takes place. As a result of these steps, we get a final blood vessels segmented image.

\section{1) Preprocessing}

This technique uses unsharp masking for preprocessing. Unsharp masking works by using a blurred version of image. This blurred image is then subtracted from original image to detect the corners and edges using (3).

$$
f_{h p}(x, y)=f(x, y)-f_{l p}(x, y)
$$

where $f(x, y)$ is the original image and $f_{l p}(x, y)$ is the blurred edition of image. Fig. 6 shows the original retinal image and unsharped masked image.

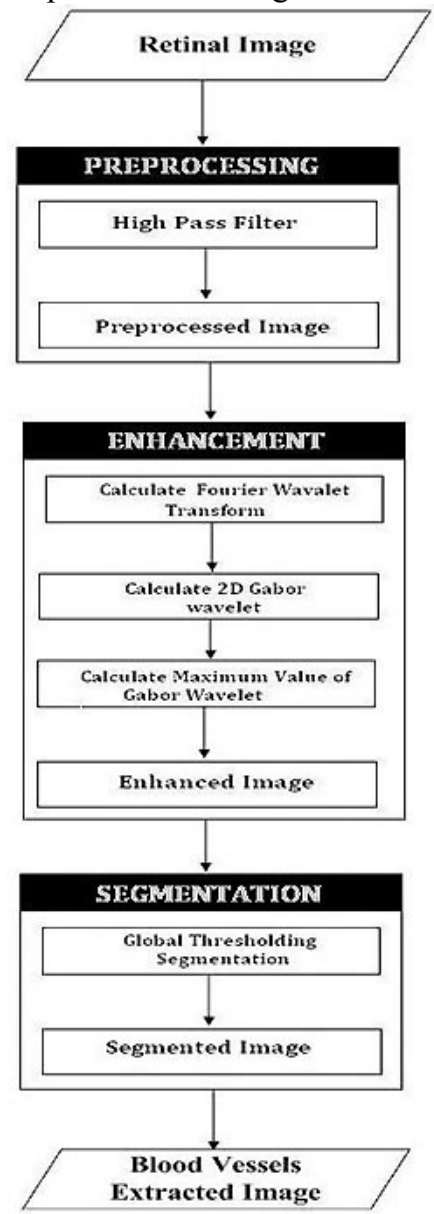

Fig. 5. Flow chart of gabor wavelet and global threshold technique 


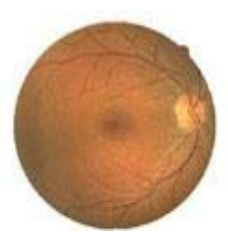

(a)

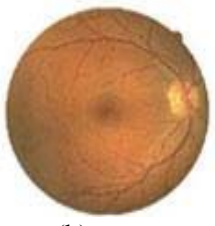

(b)
Fig. 6. Preprocessing: (a) Original image (b) Unsharped image

2) Blood vessel enhancement

Enhancement process in technique 2 uses Gabor wavelet transform [10]-[11].

1) The continuous wavelet transform $T_{\psi}(b, \theta, a)$ is defined in terms of the scalar product of $f$ with the transformed wavelet $\psi_{b}, \theta, a$ using (4).

$$
\begin{aligned}
& T_{\psi}(b, \theta, a)=C_{\psi}^{-1 / 2}\left\langle\psi_{b}, \theta, a \mid f\right\rangle \\
& =C_{\psi}^{-1 / 2} a^{-1} \int \psi^{*}\left(a^{-1} r_{-\theta}(x-b)\right) f(x) d^{2} x
\end{aligned}
$$

where $C_{\psi}, \psi, b, \theta$ and $a$ denotes the normalizing constant, analyzing wavelet, the displacement vector, the rotation angle, and the scale, respectively.

2) It is easy to implement wavelet transform using the fast Fourier transform algorithm using (5).

$$
T_{\psi}(b, \theta, a)=C_{\psi}^{-1 / 2} a \int \exp (j k b) \hat{\psi}^{*}(a r-\theta k) \hat{f}(k) d^{2} k
$$

where $j=\sqrt{-1}$, and the hat (i.e. $\hat{\psi}^{*}$, and $\hat{f}$ ) denotes a Fourier transform.

3) The 2-D Gabor wavelet is defined in (6).

$$
\psi_{G}(x)=\exp \left(j k_{0} x\right) \exp \left(-1 / 2|A x|^{2}\right)
$$

where $A$ is a $2 \times 2$ diagonal matrix that defines the anisotropy of the filter and $k_{0}$ is a vector that defines the regularity of the complex exponential. [11]

4) The response with highest modulus over all $\theta$ ranging from $0^{\circ}$ up to $170^{\circ}$ is calculated for each pixel location using (7) [11].

$$
M_{\psi}(b, a)=\max _{\theta}\left|T_{\psi}(b, \theta, a)\right|
$$

Fig. 7 shows the preprocessed image and Gabor wavelet enhanced image.

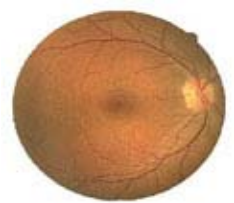

(a)

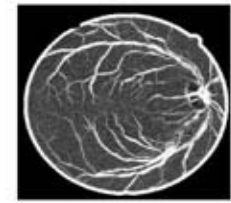

(b)
Fig. 7. Enhancement: (a) Preprocessed image (b) Gabor wavelet enhanced image

\section{3) Blood vessel segmentation}

Segmentation process in this technique has been done by global thresholding. Basic steps of global threshold segmentation are:

1) Take the Gabor wavelet enhanced image

2) Compute histogram of enhanced image

3) Find maximum occurring gray level of image

4) Calculate the threshold value
5) Convert image to binary using threshold value

Fig. 8 shows the Gabor wavelet enhanced image and segmented image.

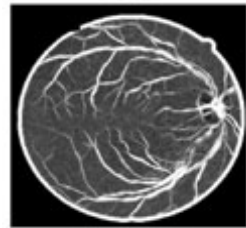

(a)

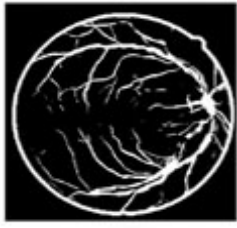

(b)
Fig. 8. Segmentation: Gabor wavelet enhanced image (b) Global threshold segmented image

\section{EXPERIMENTAL RESULTS}

For the performance evaluation of both techniques, pubicly available DRIVE (Digital Retinal Images for Vessel Extraction) and STARE (Structured Analysis of Retina) databases were used. DRIVE database contains 40 retinal images of size $768 \times 584$ pixels, eight bits per color channel. The STARE database contains 20 images of resolution $605 \times 700$ pixels, 24 bits per pixel. Both retinal image datasets (DRIVE and STARE) are divided into a test and training set and each one contains 20 images. The test set is used for measurement of performance of the vessel segmentation algorithms. There are two hand-labeling available for the 20 images of test set made by two different human observers. The manually segmented images by 1 st human observer are used as ground truth and the segmentations of set B are tested against set A, serving as a human observer reference for performance comparison truth [11], [12]. Table I summarizes the results of both techniques using DRIVE. It shows results in terms of average accuracy and standard deviation. This table clearly shows that blood vessel enhancement through Gabor wavelet and segmentation through global threshold gives better results. Same parameters are calculated for STARE database and are summarized in Table II.

TABLE I: Vessel Segmentation Results (Drive DATASET)

\begin{tabular}{|c|c|c|}
\hline Technique & $\begin{array}{c}\text { Average } \\
\text { Accuracy }\end{array}$ & $\begin{array}{c}\text { Standard } \\
\text { Deviation }\end{array}$ \\
\hline $2^{\mathrm{ND}}$ Observer & 0.9473 & 0.0048 \\
\hline Staal et. al. & 0.9441 & 0.0079 \\
\hline Soares et. al. & 0.9466 & 0.0055 \\
\hline $\begin{array}{c}\text { Gabor Wavelet and } \\
\text { Global Threshold }\end{array}$ & $\mathbf{0 . 9 5}$ & $\mathbf{0 . 0 0 5 0}$ \\
\hline $\begin{array}{c}\text { LoG filter and Adaptive } \\
\text { Threshold }\end{array}$ & $\mathbf{0 . 9 0}$ & $\mathbf{0 . 0 0 8 3}$ \\
\hline
\end{tabular}

TABLE II: VESSEL SEGMENTATION RESUlts (STARE DATASET)

\begin{tabular}{|c|c|c|}
\hline Technique & Average Accuracy & Standard Deviation \\
\hline $2^{\text {nd }}$ Observer & 0.9351 & 0.0171 \\
\hline Hoover et. al. & 0.9275 & 0.0247 \\
\hline Staal et. al & 0.9516 & 0.0329 \\
\hline Soares et. al & 0.9480 & 0.0298 \\
\hline $\begin{array}{c}\text { Gabor Wavelet } \\
\text { and Global } \\
\text { Threshold }\end{array}$ & $\mathbf{0 . 9 5 8 8}$ & $\mathbf{0 . 0 3 3 3}$ \\
\hline $\begin{array}{c}\text { LoG filter and } \\
\text { Adaptive } \\
\text { Threshold }\end{array}$ & $\mathbf{0 . 9 1 1 2}$ & $\mathbf{0 . 0 2 4 1}$ \\
\hline
\end{tabular}


Fig. 9 shows the result of both techniques on different input retinal images.

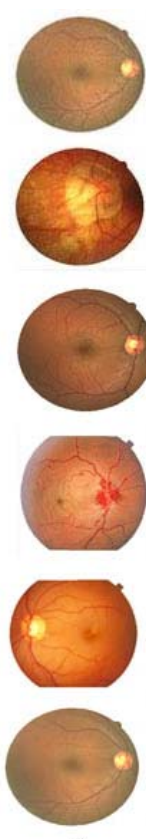

(a)
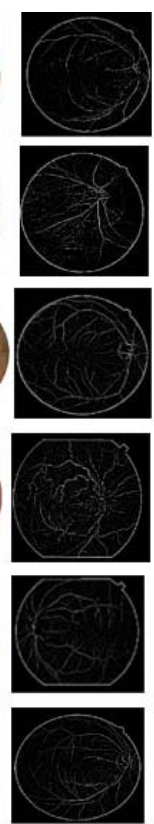

(b)
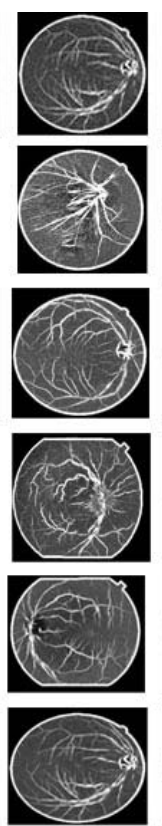

(c)
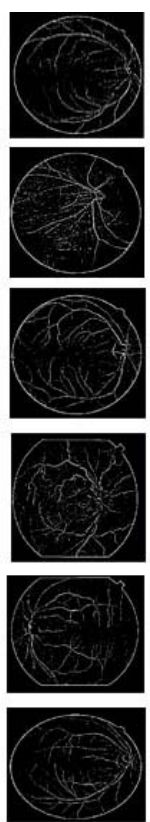

(d)
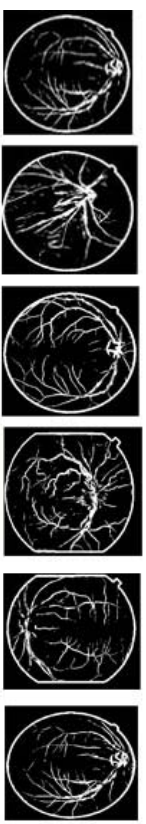

(e)
Fig. 9. Experimental Results: (a) Original Images from DRIVE and STARE, (b) Enhanced images using technique 1,

(c) Enhanced images using technique 2, (d) Segmented images using technique 1, (e) Segmented images using technique 2.

\section{CONCLUSION}

The two computerized methods for retinal blood vessel enhancement and segmentation are compared in this paper. The two techniques are evaluated using DRIVE and STARE databases of retinal images. It is concluded that Gabor wavelet and global thresholding technique is better for the vessel enhancement and segmentation respectively as the Gabor wavelet enhances the vessel as well as de noises the image and global thresholding segment all sizes of foreground vessels whether small or large. The other technique using LoG filter for enhancement and adaptive thresholding for segmentation gives less accurate results and the visibility of vessels is less in this technique.

\section{REFERENCES}

[1] C. Perra, M. Petrou, and Daniele D. Giusto, "Retinal image segmentation by watersheds," IAPR Workshop on Machine Vision Applications, November 2000.
[2] C. Wu and G. Agam, "Probabilistic Retinal Vessel Segmentation," in Proceedings of the SPIE (The International Society for Optical Engineering), vol. 6512, March 2007.

[3] C. Wu, G. Agam, and Peter Stanchev, "A hybrid filtering approach to retinal vessel segmentation," IEEE International Symposium on Biomedical Imaging, pp. 604-607, April 2007.

[4] M. Al-Rawi, M. Qutaishat, and M. Arrar, "An improved matched filter for blood vessel detection of digital retinal images," Computers in Biology and Medicine, vol. 37, 2007.

[5] Q. Li, J. You, L. Zhang, and P. Bhattacharya, "Automated retinal vessel segmentation using gabor filters and scale multiplication," IPCV 2006, pp. 22-28.

[6] I. Liu and Y. Sun, "Recursive tracking of vascular networks in angiograms based on the detection-deletion scheme," IEEE Trans. Med. Imag., vol. 12, no. 2, pp. 334-341, 1993.

[7] L. Zhou, M. S. Rzeszotarski, L. J. Singerman, and J. M. Chokreff, "The detection and quantification of retinopathy using digital angiograms," IEEE Trans. Med. Imag., vol. 13, no. 4, pp. 619-626, 1994.

[8] T. McInerney and D. Terzopoulos, "T-snakes: Topology adaptive snakes," Medical. Image Analysis, vol. 4, pp. 73-91, 2000.

[9] R. Toledo, X. Orriols, X. Binefa, P. Radeva, J. Vitri, and J. Villanueva, "Tracking of elongated structures using statistical snakes," IEEE Comput. Soc. Conf. Comput. Vision Pattern Recog, CVPR, 2000.

[10] D. J. Cornforth, H. J. Jelinek, J. J. G. Leandro, J. V. B. Soares, R. M Cesar, Jr., M. J.Cree, P. Mitchell, and T. Bossomaier, "Development of retinal blood vessel segmentation methodology using wavelet transforms for assessment of diabetic retinopathy," Journal published in Complexity International, vol. 11, pp. 50-61, 2005.

[11] J. V. B. Soares, J. J. G. Leandro, R. M. Cesar Jr., H. F. Jelinek, and M. J. Cree, "Retinal vessel segmentation using the 2-D gabor wavelet and supervised classification," IEEE Transactions on Medical Imaging, vol. 25, no. 9, pp. 1214-1222, September 2006.

[12] A. Hoover, V. Kouznetsova, and M. Goldbaum, "Locating blood vessels in retinal images by piecewise threshold probing of a matched filter response," IEEE Trans. Med. Imag, vol. 19, no. 3, pp. 203-211, 2000 .

Safia Shabbir is a student of final year of Masters in software engineering at Bahria University, Pakistan. She has done her Bachelor's in Software Engineering from Fatima Jinnah Women University Rawalpindi, Pakistan. Her research interests includes document analysis, biometric, medical image processing.

Anam Tariq is a visiting lecturer in Department of Computer \& Software Engineering, Bahria University Islamabad, Pakistan. She has done her Masters in Computer Engineering from NUST in 2013. She has wide variety of research articles and journals to her credit. Her current research interests focus on medical image processing, biometrics and pattern recognition.

M. Usman Akram is an assistant professor in Department of Computer Engineering, NUST, Pakistan. He has done his $\mathrm{PhD}$ in Computer Engineering from NUST in 2012. He received several awards for his research and teaching excellence. His current research interests focus on medical image processing, biometrics, pattern recognition and machine learning and his current research findings appear in wide variety of journals. 